Pensar la Publicidad

ISSN-e: 1989-5143

\title{
El legado de la Bauhaus en la identidad visual gráfica de las organizaciones: ideología de lo plano y flat design
}

\author{
Antonio Raúl Fernández Rincón ${ }^{1}$
}

Recibido: 15 de abril de 2019/ Aceptado: 07 de junio de 2019

Resumen. Este artículo estudia las posibles influencias de la Bauhaus en la concepción actual del diseño gráfico, concretamente en el ámbito de la identidad visual gráfica de las organizaciones. A partir de una revisión bibliográfica inicial y a través de un análisis cualitativo de las marcas gráficas más reputadas a nivel mundial, tratamos de establecer conexiones entre las propuestas de la Escuela y la tendencia actual en diseño gráfico conocida como flat design. A pesar de situarse en contextos sociales radicalmente diferentes, observamos coincidencias significativas en el origen de ambos fenómenos y en la articulación de las propuestas.

Palabras clave: Bauhaus; diseño gráfico; marcas; identidad visual gráfica; flat design.

\section{[en] The Legacy of the Bauhaus in the Graphic Visual Identity of Organizations: Ideology of Flatness and Flat Design}

\begin{abstract}
This article studies the possible influences of the Bauhaus in the current conception of graphic design, specifically in the field of graphic visual identity of organizations. Based on an initial literature review and through a qualitative analysis of the most reputable graphic brands worldwide, we try to establish connections between the proposals of the School and the current trend in graphic design, known as flat design. In spite of being situated in radically different social contexts, we observed significant coincidences in the origin of both phenomena and in the articulation of the proposals.
\end{abstract}

Keywords: Bauhaus; Graphic Design; Brands; Graphic Visual Identity; Flat Design

Sumario. 1. Introducción. 2. Marcas e identidad. 3. El diseño en la Bauhaus: forma, tipografía y color. 4. Esqueumorfismo y Flat Design. 5. Descripción metodológica. 6. Resultados. 7. Conclusiones. 8. Referencias bibliográficas.

Cómo citar: Fernández Rincón, A.R. (2019). El legado de la Bauhaus en la identidad visual gráfica de las organizaciones: ideología de lo plano y flat design. Revista Pensar en la Publicidad, 13, 65-85.

\section{Introducción}

Cien años después de su fundación en Weimar, la Bauhaus sigue siendo un concepto de referencia que sintetiza un proceso de modernización radical del diseño y la

\footnotetext{
$1 \quad$ Universidad de Murcia

antonioraul.fernandez@um.es
} 
vida. Sus escasos catorce años de vida fueron suficientes para dejar una impronta continuamente revisada y discutida acerca de los que se denomina, a veces de forma excesivamente trivial, «estilo universal». La reforma pedagógica que inició Walter Gropius en 1919, unida al legado de maestros de la época que pasaron por la escuela, han tenido un amplio eco dentro y fuera de Alemania. Maestros de la Bauhaus como Wasily Kandinsky, Johannes Itten, Lyonnel Feinninger, Paul Klee, Oskar Schlemmer, Josef Albers, László Moholy-Nagy, Herbert Bayer, Ludwig Mies van der Rohe o el propio Gropius, han influido desde entonces en la propia pedagogía del arte, en los estudios de los materiales y en los análisis elementales de colores y formas.

El programa de la Bauhaus se parece a los planes de estudios de muchas de las escuelas de arte reformadas ya antes de la guerra: el alumno debía ser instruido en artesanía, en dibujo y en ciencia. La novedad de Gropius era el subordinar la escuela a una meta: el edifico levantado colectivamente, al que todos los oficios debían contribuir (Droste: 1993: 22).

La contribución de los diferentes oficios a ese «edificio colectivo» que imaginó Gropius, ha sido y sigue siendo extensamente tratada en la literatura científica. Entre los oficios que quizá han obtenido menor protagonismo en este sentido se sitúa el diseño gráfico y más concretamente el ámbito relacionado con el diseño de la identidad visual de las organizaciones.

Este estudio trata de conectar los aprendizajes, descubrimientos y propuestas de la Bauhaus en materia de forma, color y tipografía, con tendencias recientes en la construcción de las marcas gráficas, lo que denominamos identidad visual corporativa o identidad visual gráfica.

La intención se sitúa, por tanto, en conocer cómo las propuestas de la Escuela podrían encajar con determinados estilos gráficos y formas de expresión, cien años después. Se trata así de explorar la supuesta vigencia de los conceptos y modos de hacer de la Bauhaus en el diseño de la identidad actual.

El valor de este estudio no solo conecta con la pretensión de revivir las enseñanzas de la Bauhaus en sus cien años de vida, a nivel más pragmático, también se une a la relevancia que a día de hoy toman los procesos de creación y gestión de la identidad en las organizaciones. En un mercado saturado, cambiante y convulso, la identidad de las empresas es altamente relevante porque comprende un conjunto de características (atributos) que permiten diferenciarla de otras organizaciones, independientemente de cómo sean percibidas en su contexto exterior (Sanz de la Tajada, 1996: 32). La marca constituye hoy un sistema sígnico encargado de transmitir a los universos paralelos y circundantes todos los rasgos de la personalidad de la corporación; la marca se ha convertido en una auténtica «seña de identidad» al servicio de la imagen - corporativa- de la empresa. (Contreras y San Nicolás, 2001: 118). De igual forma, resulta interesante valorar en qué medida los presupuestos de la Bauhaus en el terreno de la identidad, encajan con las necesidades de las marcas actuales en el contexto de la digitalización y los entornos multimedia.

En línea con estas intenciones, tratamos de demostrar cómo algunas de los principios básicos de la forma, la tipografía y el color en la Bauhaus, perviven hoy en el ámbito del diseño gráfico, concretamente en lo que se refiere a la creación de la 
identidad visual de las organizaciones y la tendencia conocida como flat design. Para ello se realizará una revisión de aspectos clave de teoría del diseño de cara a crear un marco conceptual en torno al objeto de estudio así como un apropiado contexto sobre las cuestiones que afectan a la enseñanza de esta disciplina en la Bauhaus mediante una revisión crítica usando el método histórico. Una vez establecido este marco teórico, se procederá al estudio de caso a través del análisis de la evolución en la identidad visual de organizaciones con mejor reputación.

\section{Marcas e identidad}

El primer escollo al que se enfrenta cualquier estudio sobre la marca y su identidad, se refiere a la confusión terminológica que pervive sobre el fenómeno. La amplitud de términos y la disparidad de nociones que se emplean para referirse al mismo fenómeno, no contribuye a unificar marcos teóricos y metodológicos de referencia, ni a consolidar procesos funcionales para las empresas (imagen corporativa; identidad corporativa, identidad visual; marca gráfica; identidad visual gráfica; identificador corporativo). En líneas generales, tal y como afirma Currás-Pérez (2010), encontramos dos vertientes enfrentadas: por un lado, los trabajos que conciben el concepto de identidad corporativa como un constructo que encierra una noción profunda y esencial de lo que es la organización, de su ethos, de aquello que la caracteriza y le confiere singularidad. Por otro, los que aportan una visión de la identidad que se ciñe exclusivamente a los modos o mecanismos de expresión (comportamiento, comunicación, simbolismo) de los que dispone la organización para proyectar su personalidad corporativa.

La noción de imagen corporativa, a diferencia de la identidad corporativa, descansa sobre un mayor consenso ya que normalmente se asocia con la percepción, es decir, la suma de creencias, experiencias, sentimientos o información (Kotler y Armstrong, 1991) que los consumidores y/o stakeholders (Balmer, 2001) poseen de una organización o marca. Para Mínguez (2000), la imagen corporativa está relacionada con las operaciones cuyo fin es crear determinadas impresiones en los públicos, siendo por tanto la imagen un reflejo de la identidad cuyo destino final es determinar la actitud de los públicos en un sentido que sea positivo para la empresa. La identidad corporativa, por otra parte, está constituida en sentido amplio por los rasgos esenciales que hacen a cada organización diferente de las demás. Estaría determinada además, por cuatro factores: el comportamiento corporativo, la cultura corporativa, la identidad visual y la comunicación corporativa.

En este sentido, Rodríguez González (2005) propone una herramienta de análisis teórico formal que sirva para evaluar cualquier trabajo en el terreno de la imagen corporativa o institucional. El autor propone una taxonomía de lo que denomina «imágenes logotípicas» a partir de los tratados más recurrentes en este ámbito: Punto y línea de Vasily Kandinski en 1926; La sintaxis de la imagen de Dondis en 1972; Fundamentos del diseño de Wucius Wong, 1977, así como las obras de Rudolf Arnheim, Gaetano Kanizsa o Rosa Puentes. La terminología más empleada hoy en día para referirse a las variedades formales de la identidad, es la que distingue entre logotipo, isologo, isotipo e imagotipo ${ }^{2}$.

2 Clasificación empleada por la consultora de branding, Summa. Ha sido empleada en el presente estudio. Recurso electrónico disponible en: https://bit.ly/2X2ELUP 
Para Villafañe (1998: 116-123) los principios generales de la imagen corporativa son su carácter normativo y su intención integradora. De ellos derivarían el resto de principios específicos de la identidad visual. La norma, por tanto, homogeneiza y determina las aplicaciones corporativas, mientras que la integración facilita la construcción de la imagen de una empresa en la mente de sus públicos a partir de la mancomunión de un conjunto de elementos -identidad visual + cultura + comunicación- los cuales trabajan unidos hacia la consecución de un mismo objetivo.

De estos principios generales, emanan los cuatro principios específicos de la identidad visual (Costa, 1987: 91-104).

- Principio simbólico: Los signos de identidad visual traducen simbólicamente a formas, colores y tipografías aquello que no pertenece a la realidad material (atributos o facultades psicológicas).

- Principio estructural o gestáltico: Se trata de diseñar «estructuras visuales» capaces de transmitir un «todo», a través de una identidad integrada y normalizada.

- Principio sinérgico: Este principio preside el desarrollo del manual de normas de identidad visual velando por el tono armónico de todas las aplicaciones (logotipo, símbolos, logosímbolo, colores, tipografías, tono, estilo, formatos, recursos, etc.)

- Principio de universalidad. Un programa de identidad ha de ser duradero, evitando planteamientos coyunturales más o menos efímeros. Al mismo tiempo debe procurarse la proyección de la identidad de la empresa en diferentes soportes y asegurar el uso de códigos culturales, anclados en el ámbito de actuación de la empresa o institución.

Más allá de su significación y en línea con Chaves y Bellucia (2003: 16) lo relevante es la función que ejerce y no tanto la denominación empleada. Adrian Frutiger hablaba de signos-marca como elementos imprescindibles en la economía moderna.

En la pugna competitiva siempre creciente de la economía de lo visualmente anónimo está fatalmente condenado. El comprador ya no se fía del producto que carece de nombre ni del servicio sin personalidad conocida. Para conseguir hoy una posición en el mercado y lo que es más, para conservarla se hace cada vez más preciso el crearse una imagen de la propia identidad (Frutiger, 2007:260)

El identificador corporativo o marca gráfica, el signo visual de cualquier tipo (logotipo, símbolo, monograma, mascota, etc.) tiene la función de individualizar a una entidad, al igual que un nombre propio. El identificador visual -sea o no de naturaleza verbal como lo es el logotipo-constituye una suerte de sinónimo visual del nombre. Un identificador no verbal abstracto (como el símbolo de Mercedes Benz) o icónico (como el de Apple) cumple la misma función que los respectivos nombres (Mercedes Benz y Apple) y sus logotipos.

Entre los principales retos a los que se enfrenta la identidad de las organizaciones en la actualidad, se encuentra la necesaria adaptación al nuevo ecosistema mediático, provocado por la irrupción masiva de las tecnologías de la información y la 
comunicación. No es el único reto pero sí el más relevante para nuestro estudio. La multiplicación de escenarios, soportes y pantallas en las que deben habitar las marcas y sus identificadores, obliga a una revisión profunda de los procedimientos de creación y gestión, con vistas a lograr la homogeneidad y coherencia gráfica que tradicionalmente se exige a estos elementos. En este sentido, uno de los conceptos que más preocupan a los creadores y gestores de marcas gráficas es el de «adaptabilidad»

En el ámbito de la identidad visual, los denominados logotipos responsive o adaptativos configuran una nueva tendencia que afecta principalmente a grandes marcas. El objetivo es diseñar logotipos que sean capaces de adaptarse dependiendo del dispositivo desde el que se visualizan. Diseñar posibles variaciones en la identidad no es algo reciente, tradicionalmente los manuales de identidad corporativa ya incorporan posibles eventualidades donde la marca gráfica debe alterar su disposición y/o forma para adaptarse a soportes o materiales que pudieran dificultar su correcta visión.

Con la multiplicación de soportes digitales desde los que acceder a la información, el diseño de los logos ha tenido que contemplar en muchos casos, posibles variaciones constructivas que siempre van por el camino de la simplificación y la reducción a la mínima expresión gráfica reconocible. El especialista en diseño digital Joe Harrison, lo define como «Scalable Branding» (fig. 1). El concepto, según el autor, es la evolución del branding desde una posición fija e inamovible hacia un sistema flexible y contextual.

Fig. 1. Scalable branding.

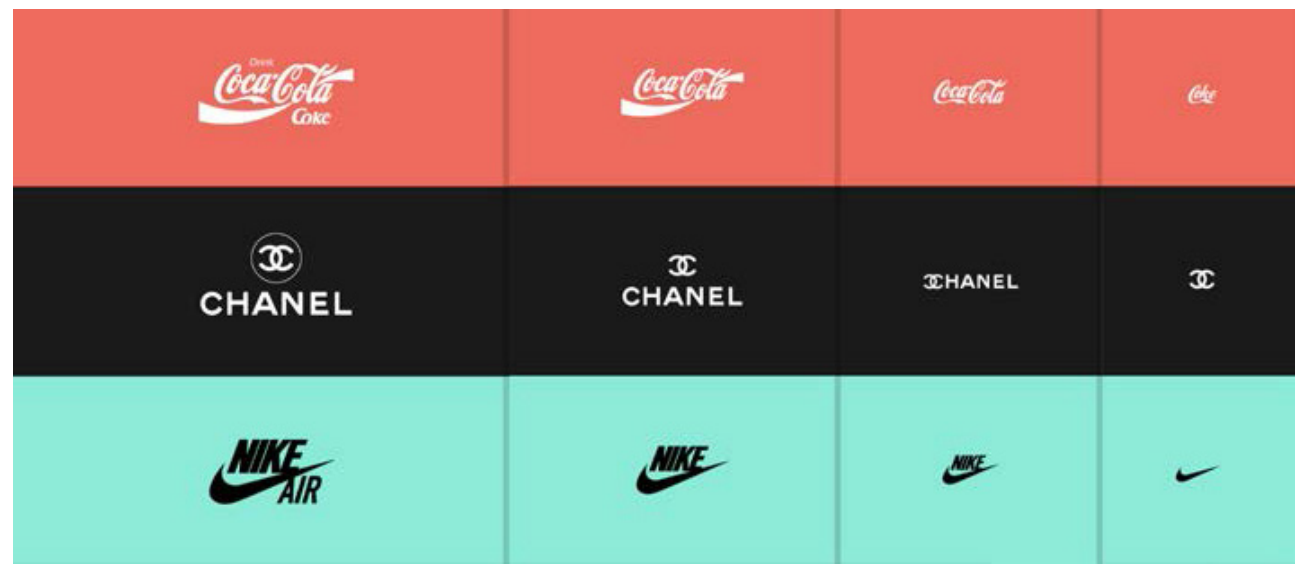

Fuente: http://www.joeharrison.co.uk/projects/responsivelogos

En el ámbito del diseño web, la construcción responsive o adaptativa es una técnica que se orienta hacia la homogeneidad en la visualización de los contenidos web en diferentes dispositivos. De esta forma, se trata de situar los elementos de forma que se adapten a las diferentes medidas sobre las que trabajan los distintos soportes: ordenador de sobremesa, portátiles, tablets, smarthphones y smartwatches. 


\section{El diseño en la Bauhaus: forma, tipografía y color}

La oferta formativa de la Bauhaus se integraba por diferentes talleres que fueron tomando forma según se encontraban maestros capacitados, ya que solo ellos estaban cualificados y tenían derecho a enseñar a los aprendices. Se integraron en la escuela diferentes talleres entre los que destacaron: el taller textil, de carpintería, pintura en vidrio y pintura mural, alfarería, cerámica, taller del metal, de mobiliario, de escultura y talla, teatro, encuadernación, además de la formación en color y forma. Además de estos talleres, surgieron áreas de trabajo e iniciativas que además de servir de apoyo a la docencia, proporcionaban servicios internos y externos. En este sentido destacamos la imprenta gráfica, la editorial, la revista Bauhaus y el taller de impresión y publicidad.

- La imprenta gráfica, dirigida por Lyonel Feininger se encargaba de editar libros y cartapacios para la escuela y sus maestros. Además, a instancias de Gropius funcionaba como un taller independiente que aceptaba trabajos de clientes ajenos a la escuela.

- La editorial «Bauhaus Munich-Berlín» fue creada en 1922 para fortalecer la independencia económica de la Bauhaus y ayudar a difundir su mensaje.

- La revista Bauhaus se publicó con periodicidad cuatrimestral desde finales de 1926 hasta 1931 para dar información sobre la actividad de la escuela, los nuevos productos y los contratos realizados. Estaba dirigida por Herbert Bayer.

En el ámbito del diseño gráfico, tanto la imprenta como la editorial y la revista se convirtieron en espacios reales para la materialización de los conceptos teóricos propuestos por los maestros y realizados por estos mismos o sus aprendices. La enseñanza del diseño en la Bauhaus se fundamentaba básicamente en el dominio de tres aspectos esenciales: la forma, la tipografía y el color.

\subsection{La forma}

Para comprender los fundamentos de la forma en la Bauhaus, hay que retroceder hasta sus antecedentes ideológicos. La ideología de la Escuela se situó entre la frontal oposición al maquinismo -producto de la Revolución Industrial y procedente fundamentalmente de Inglaterra a través de John Ruskin y William Morris- y el rechazo hacia el art nouveau. En ambos casos las posibilidades estéticas que ofrece la producción mediante máquinas, actuaban en detrimento del trabajo manual. Para artistas como Herman Muthesius o Henry Van de Velde el recubrimiento de los objetos industriales era una equivocación repulsiva que debía combatirse con la objetivación y la limpieza ornamental. El ornamento gótico es un lastre o costra que impide desarrollar y mostrar la finalidad del objeto. En este sentido, se reivindica la fuerza de la línea en el sentido constructivista y en contraposición a su utilización con fines decorativos, tan recurrente en el art nouveau. Muthesius había sido enviado a Inglaterra en 1986 por el gobierno prusiano, como «espía del gusto» para estudiar el influyente movimiento de las arts and crafts en Inglaterra, teniendo en cuenta principalmente los valores de artesanía y economía como factores de un buen diseño. En 1907 fundó la «Deutscher Werkbund», una asociación mixta para la promoción de los productos artesanos alemanes. El principio fundacional de la asociación era el 
diseño racional que eliminaba la ornamentación y la decoración superflua, enfatizando el funcionalismo. Esta sencillez repercutía en una mayor estandarización y eficacia productiva que se transformaba en un incremento de la calidad y un mayor ahorro para el usuario y el fabricante. Junto a Muthesius se situaron Friedrich Naumann, Joost Schmidt y Peter Behrens, este último poco después fue contratado por la compañía AEG como diseñador jefe, dando un estilo inconfundible a los materiales promocionales de sus diseños para teléfonos, ventiladores o farolas.

Desde una perspectiva historiográfica en el contexto de la Bauhaus y las primeras vanguardias resulta inevitable hacer referencia a la obra Planilandia, una novela de muchas dimensiones (Abbott, 1999), editada por primera vez en 1884 configura una obra seminal para los movimientos artísticos de principio de siglo XX que comienzan a apostar por la superficie plana y una realidad abstracta alejada de lo puramente mimético. En este sentido y ya en el seno de la propia escuela cabe incluir las obras de profesores como Paul Klee o Wasily Kandinsky. Klee publicó las enseñanzas en el aula hasta 1931 en Cuadernos pedagógicos y Kandinsky hizo lo propio en Punto y Linea sobre el Plano, de 1926.

El desarrollo de la Bauhaus estuvo marcado en cierta medida por las discrepancias y la marcada personalidad de algunos artistas que se integraron en el cuerpo docente. Esto dejó entrever en ocasiones miradas discrepantes que impiden hoy la consideración de un «estilo Bauhaus» como algo cerrado y unívoco. En este sentido, Wick, (1982: 235239), señala los años de Oskar Schlemmer (1888-1943) en la Bauhaus como ejemplo del escepticismo de algunos miembros frente a las posiciones dogmáticas de la Escuela y la tensión constante entre lo clásico y lo romántico. La ruptura de la «ideología de lo plano», bajo la influencia del constructivismo abstracto, en sus «Figuras en el Espacio» (1925), provocó molestias en otros artistas que veían una traición a lo moderno y una vuelta al conservadurismo. El objetivo había sido, según el autor, una ganancia en comprensibilidad y clasicismo. A pesar de ello, Schlemmer desarrolló en 1922 el conocido emblema oficial de la Bauhaus (fig. 2) que muestra una cabeza humana de influencia claramente constructivista. Uno de las escasas concesiones del autor a la ideología dominante de la Escuela respecto al ángulo recto y el imperativo del cubo.

Fig. 2. Emblemas de la Bauhaus diseñados por Karl Peter Röhl (izquierda) y Oskar Schlemmer (centro).
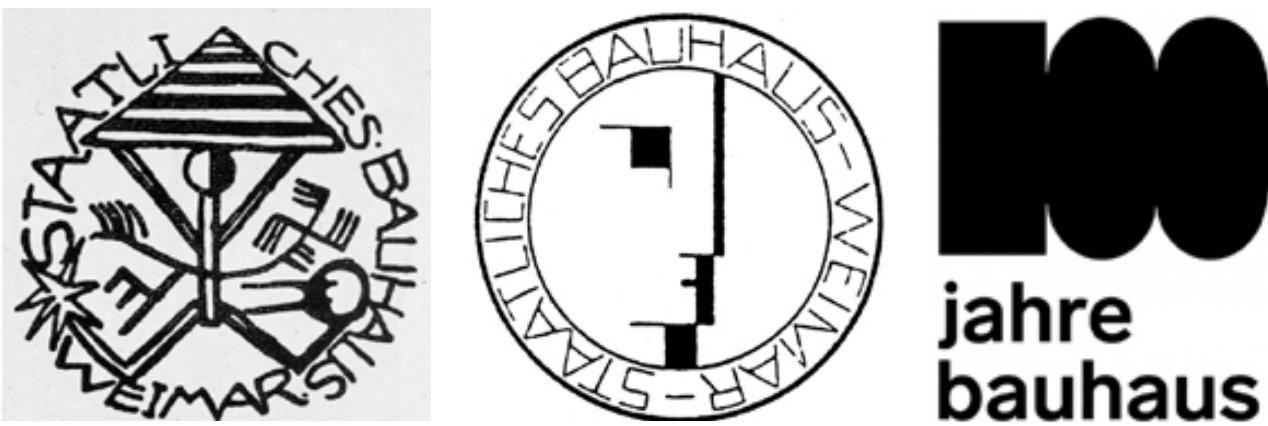

Fuente: (Whitford: 1991:119). A la derecha, la identidad creada por la agencia Stan Hema ${ }^{3}$ con motivo del centenario en 2019.

Recurso electrónico disponible en: https://bit.ly/2CpgEm7 
El movimiento De Stijl fue constituido en Países Bajos en 1917 por un grupo de pintores, diseñadores y arquitectos, entre los que destacaron: Piet Mondrian, Bart van der Leck o Theo van Doesburg, este último, fundador y principal inspirador. Las ideas de este grupo de creadores se encuadraban dentro del Neoplasticismo, una corriente que como el Purismo, el suprematismo o el constructivismo, surge después de la Primera Guerra Mundial a partir del cubismo. El ideal del neoplasticismo suponía una reivindicación de la abstracción progresiva donde las formas se reducen a líneas rectas y los colores a los tres primarios: el blanco y el negro. No existe profundidad, ni referencias figurativas. Es arte puro: línea y color, unidas en un mismo espacio, rechaza cualquier motivo reconocible, que imite la realidad. El arte forma ya parte de la vida real, así que no es necesaria su imitación.

\subsection{La tipografía}

Los antecedentes del estilo tipográfico implantado por la Bauhaus se remontan al siglo XIX y el impacto de la industrialización en las artes gráficas. Las líneas gruesas en los tipos favorecían el contraste del trazo y soportaban mejor las exigencias de la impresión. Las denominadas Gothic en Estados Unidos o Grotescas en Europa fueron las primeras familias tipográficas que prescindían de remates (sans serif). Se trataba de tipos pesados y rígidos empleados comúnmente para títulos. En España se denominaron letras de palo seco. Otras referencias obligadas en este sentido pueden encontrase en los trabajos de Edward Johnston para el Metro de Londres y la incursión del arquitecto y diseñador escocés Charles Rennie Mackintosh en el ámbito de la tipografía. La tipografía Johnston Sans que con leves modificaciones sigue empleándose hoy en día, supuso una importante innovación al estar desprovista de remates y adornos propios del estilo victoriano. Era una tipografía geométrica que facilitaba la rápida lectura de los paneles en el denominado Tube londinense. La tipografía ITC Rennie Mackintosh, comercializada aún hoy en día, podría entenderse como la materialización tipográfica del estilo -orgánico al principio y geométrico después- del que es considerado uno de los máximos exponentes del art nouveau. La tipografía creada por el artista contiene formas geométricas sin remates y con leves adornos, también geométricos, en algunas grafías.

En los primeros años de la Bauhaus se había experimentado con los expresivo y lo pintoresco, lo que en ocasiones dificultaba seriamente la legibilidad. En 1923 Moholy-Nagy sentaba las bases del dogma tipográfico que artistas como Josef Albers, Joost Schmidt o el propio Herbert Bayer llevarían a su máximo extremo.

La tipografía es un instrumento de comunicación. Debe ser una comunicación clara y eficaz... La legibilidad de la comunicación nunca puede sufrir bajo una estética admitida a priori, Los tipos de letra nunca deben ser forzados en una forma predeterminada (Moholy-Nagy, citado por Wick, 1982: 46).

Josef Albers diseñó en 1925 la plantilla de letras, un alfabeto fácilmente legible a distancia para anuncios y carteles que surge a partir del reduccionismo formal de Kandinsky sobre las formas básicas del cuadrado, el triángulo y el círculo.

El desconocido profesor de la Escuela, Joost Schmidt, destacó por su compromiso pedagógico con la escritura que incluía un rechazo frontal hacia la obsesión alemana por la letra gótica, algo que llegó a calificar de «escoria cultural». Bayer fue un alumno brillante, en 1925 fue nombrado profesor de tipografía y publicidad, cargo que desempeñó 
hasta 1928, justo antes de su traslado a Berlín para trabajar como diseñador gráfico especializado en espacios expositivos, tipógrafo, pintor y fotógrafo. Creía en el carácter primitivo del diseño gráfico y ante todo de la publicidad. En su obra hacía extensivo el uso de la caja baja (minúsculas), realizaba sus diseños usando retículas sistematizadas con tipos de palo seco, legibles y de ancho uniforme para conseguir composiciones limpias y geométricas. A finales de 1925, como director de la revista Bauhaus, impuso la escritura de todos los textos en caja baja y la normalización de la impresión al sistema $\mathrm{DIN}^{4}$. Al pie de cada página aparecía la leyenda: «escribimos todo en minúscula porque así ahorramos tiempo, además; ¿por qué dos alfabetos, cuando uno es suficiente? ¿para qué escribir mayúsculas, cuando no pueden pronunciarse» (Citado por Drosde, 1993: 139).

Bayer creo la «Tipografía Universal» (fig. 3) de formas geométricas puras y depuradas a partir de las aportaciones del tipógrafo alemán Jan Tschichold, en su libro «Die Neue Typographie» (La nueva tipografía), una de las obras de referencia sobre tipografía del siglo XX. Joost Schmidt creó el «Alfabeto Elemental» (fig. 4).

Las tipografías de Bayer y Schmidt se reducían a formas simples construidas recurriendo a una rigurosa geometría. Se omitieron las mayúsculas por entender que no había diferencia entre el sonido expresado por la caja alta y la baja y por lo tanto eran innecesarias (Pelta, 2012-B). Este racionalismo constructivo, o «Tipo Universal» lejos de entroncar con el romanticismo y el naturalismo europeo, conectaba al otro lado del Atlántico con el americanismo que, entre otros aspectos, inspiraría el fordismo, el taylorismo y la ruptura con las tradiciones esclavizantes. Bayer dio a luz un nuevo lenguaje visual corporativo a partir de la regularidad formal. Una vez abandonó la escuela, aunque no puede hablarse de un rotundo éxito de sus postulados en el ámbito empresarial, sí puede hablarse una herencia casi mística que sirvió para que numerosos artistas gráficos y diseñadores del siglo XX pudieran desarrollar importantes encargos en el área corporativa. Uno de los ejemplos más sobresalientes es el diseñador estadounidense Paul Rand, con su logotipos para marcas como ABC, UPS, Westinghouse o el imperecedero anagrama diseñado para IBM (Pelta, 2012).

Fig.3. Tipografía Universal de Bayer.

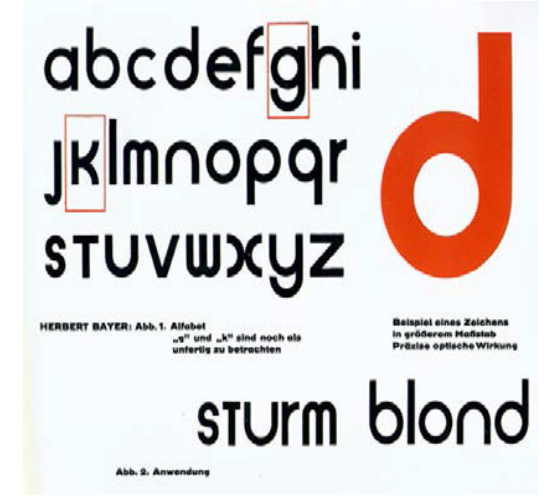

Fuente: (Droste: 1193:149)

4 (DIN) Instituto Alemán de Normalización. Organismo de normalización en Alemania que elabora estándares y normas técnicas de normalización para el comercio y la industria. En Europa se ha generalizado el uso de la norma DIN 476, como referencia a los tamaños de papel para impresión. Recurso electrónico disponible en: https://www.din.de/en 
Fig.4. Alfabeto elemental de Schimdt.

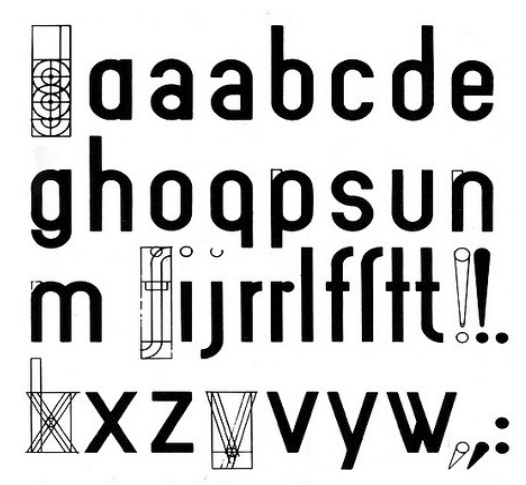

Fuente: http://www.tipografos.net

\subsection{El color}

Todos los alumnos de la Bauhaus debían superar un curso inicial (Vorkurs) para demostrar sus aptitudes de cara a la admisión definitiva. Este periodo incluía el estudio de los materiales, el análisis de antiguos maestros de la pintura y el curso básico de color y forma. Las clases fueron impartidas por Paul Klee, Wassily Kandinsky y Johannes Itten, este último, primer director del curso y que en un ensayo de 1916 afirmaba: «...las formas geométricas y los colores del espectro son las formas y los colores más simples, más sensibles y, consecuentemente, los medios de expresión más exactos en una obra de arte» (Citado por Whitford, 1984: 106). Las enseñanzas de Kandinsky en el Vorkurs consistían en el estudio del dibujo analítico y el estudio del color y la forma. El color y la forma se estudiaban al principio de manera aislada para después relacionarlos con el fondo, entendido este como el plano en el que aparecen todas las imágenes en dos dimensiones.

Tanto Itten como Kandinsky y Klee, establecieron en sus teorías numerosos paralelismos entre la pintura y la música además de sus respectivas teorías sobre el punto, la línea y el color, el fenómeno más rico de la experiencia óptica.

El color para la Bauhaus era tan importante que llegó a convertirse en asignatura obligatoria en 1922, tal y como señala Vadillo (2016: 30-31)

La teoría del color en la Bauhaus resultó ser una de las inquietudes más desarrolladas por sus maestros, una de la principales preocupaciones de las distintas personalidades que impartieron enseñanzas en la escuela. (...) De hecho, en la mayoría de los objetos que diseñaron se puede apreciar el resultado de esa formación (...) Los profesores asociaban el color a propiedades espirituales, incluso sinestésicas. No obstante, aunque el código cromático general de la escuela estaba ligado a los tres colores primarios (amarillo, rojo, azul), el color no fue impartido con criterio unánime por los maestros.

Las principales aportaciones de lo maestros del color en la Bauhaus no residieron únicamente en el estudio del color desde el punto de vista científico, también en las relaciones cromáticas y los efectos que este produce. El color no se limita a lo visual, sino también a lo psicológico y lo emocional. El círculo cromático compuesto de 12 
colores (fig. 5), el libro de Itten «El arte del color» editado en 1960, o «La interacción del color» de Albers en 1963, constituyen referencias básicas en los fundamentos de la formación contemporánea sobre el color y el lenguaje visual.

Fig.5. Círculo cromático de Itten.

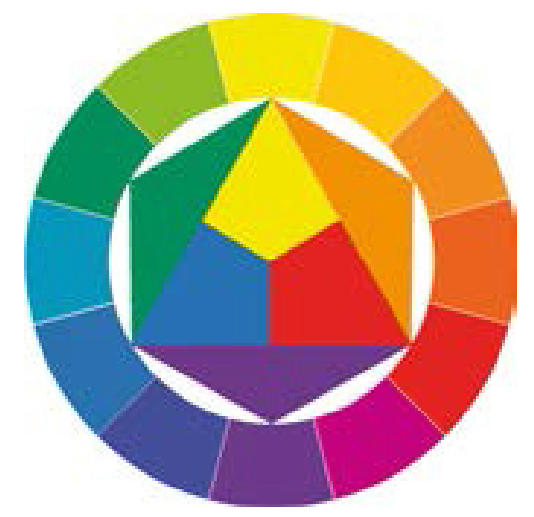

Fuente: https://bit.ly/2UcmjcFm

\section{Esqueumorfismo y Flat Design}

Esqueumorfismo es un término que proviene del griego skéuos (бкєṽos) herramienta у morphê ( raíz de la masiva introducción de los dispositivos móviles y las aplicaciones (App). En las primeras interfaces gráficas de sistemas operativos y aplicaciones móviles, para mostrarse más familiares con unos usuarios aún no habituados a esta tecnología, empezó a generalizarse un tipo de diseño que de alguna forma trataba de imitar la estética del objeto físico. Apple Computers fue una de las compañías que más impulsó el esqueumorfismo en los productos y contenidos tecnológicos durante sus primeros años de vida. Curiosamente el cofundador de la compañía Steve Jobs siempre trató de inculcar a su equipo el gusto por la simplicidad en la forma y el color. Para Mouzon (2010) la pasión por la simplicidad de Jobs era legendaria, y su estima por el diseño minimalista de la Bauhaus era inmenso, pero mientras que la Bauhaus es amada solo por los expertos en diseño, los productos de Apple son amados por las masas de todo el mundo. Apple ofreció en sus productos una interfaz adorable que hacía a las personas sentirse cómodas con sus máquinas ya que estaban más familiarizadas con su estética interna. Quería que los píxeles contaran una historia al usuario que las máquinas fueran alegóricas y amigables. El ícono del calendario se parece a un calendario de papel, el de la impresora parece una impresora y la papelera es casi una papelera real. Susan Kare fue la responsable del primer sistema de iconos para el Macintosh 128K, lanzado por la compañía en 1984.

Hasta ese momento, todo control de un ordenador se hacía escribiendo líneas de comando, y la introducción de la interfaz gráfica constituyó un avance significativo en el intento de Apple para acercar la computadora a las personas (Molina Ruiz, 2010: 13). 
El esqueumorfismo ayudó a toda una generación de usuarios a familiarizarse con las nuevas tecnologías pero también comenzó a generar una cantidad de información en el escritorio que pronto se mostró como innecesaria. Sobre todo para nuevas generaciones de usuarios alfabetizados en las tecnologías que no han conocido un mundo sin ella ${ }^{5}$. En 2007, la revista Forbes ${ }^{6}$ anunció de forma quizá algo precipitada, la muerte del esqueumorfismo a favor de un «nuevo» concepto gráfico, el diseño plano (flat design). Microsoft presentó en 2012 su sistema operativo (Windows 8) con una importante renovación gráfica de la interfaz. Destacó su claridad visual y el empleo de mosaicos de color plano que ofrecen datos escritos y visuales, sustituyendo a la iconografía de anteriores versiones. Apple por su parte, presentó su sistema operativo (iOS 7) también con este nuevo aspecto en septiembre de 2013. Gran parte de los bordes biselados y metálicos, los degradados, las sombras y los efectos tridimensionales de la interfaz había sido sustituida por un diseño sobrio, de colores y formas planas, sin profundidad y sin atisbo de realismo en los elementos gráficos.

El término flat design (diseño plano) fue popularizado en 2012 por Allan Grinshtein, en su post «The Flat Design Era». Grinshtein explica que las interfaces elegantes son las que tienen el mayor impacto con la menor cantidad de elementos. La idea es que una interfaz minimalista puede adaptarse mejor a su función en comparación con una más compleja y ornamentada. El germen de esta ideología puede encontrarse en los visionarios trabajos de Alan Kay en el Xerox PARC (Palo Alto Research Center) durante la década de los setenta. Kay enfocó el trabajo de su equipo en el desarrollo de programas fáciles de usar para niños. A través del software Smalltalk dio lugar a un estilo simplificado e intuitivo en las interfaces de usuario (GUIs). Los niños podían manipular objetos en la pantalla con sus manos y usar iconos para realizar acciones concretas. Para Barnes (2007), las aportaciones de Kay encuentran su fundamentación en los trabajos de psicólogos como Piaget y Bruner, este último enfocado en el estudio de los procesos cognitivos que realizan los niños en la toma de decisiones. Años después, las propuestas de Kay y su equipo sirvieron de inspiración para grandes compañías tecnológicas como Apple Computer o Microsoft.

A partir de Cousins (2013) y Cao, Zieba, Ellis, y Stryjewski (2015), podemos establecer 5 principios básicos del concepto flat design.

- No contiene efectos añadidos. Emplea un estilo bidimensional. Funciona sin adornos: sombras, biselados, estampados, degradados u otras herramientas que agregan profundidad. Los elementos carecen de bordes o sombras difuminadas. No se busca el realismo, se basa en un claro sentido de jerarquía en el diseño y la ubicación de los elementos para facilitar el entendimiento y la interacción en diferentes pantallas.

- Es simple e intuitivo. Utiliza muchos elementos de interfaz de usuario simples, como botones e iconos. A menudo suelen ser rectángulos, círculos o cuadrados con bordes definidos. Cada elemento debe ser simple y fácil, la interacción debe ser intuitiva. No debe confundirse elementos simples con diseño simple, los conceptos de diseño plano pueden ser tan complejos como cualquier otro tipo de esquema de diseño.

Recurso electrónico disponible en: https://bit.ly/2cQMVU8

Recurso electrónico disponible en: https://bit.ly/2UpHAOk 
- Otorga una alta importancia a la tipografía. La tipografía debe mantenerse acorde con la naturaleza simple de los elementos del diseño plano, una fuente muy ornamentada resulta extraña en comparación con un diseño simple. Predomina el uso de familias tipográficas sans-serif (Helvética, Frutiger, Franklin Gothic, Bebas, Gill, Century, Din, Futura, Gotham, Verdana, Calibri, Tahoma) en sus múltiples variantes y admitiendo toques inesperados y originales que no tomen excesivo protagonismo en el diseño general. El tipo también debe indicar a los usuarios cómo usar el diseño. Para titulares predomina el uso de la caja alta (mayúsculas).

- Emplea colores planos. Las paletas de colores de diseño plano suelen ser mucho más brillantes y coloridas. Los tonos tienden a ser más vibrantes y más puros de la rueda de colores, sin matices. Los colores primarios y secundarios son más usuales aunque los colores retro o pastel como el salmón, el púrpura o mezclas de verde azul, son también muy populares. Sin embargo, limitar un esquema de color no significa que deba diseñar en blanco y negro o con un solo color. La idea es utilizar solo los colores necesarios para representar con precisión el diseño y crear jerarquía en los contenidos. El espacio blanco es un elemento esencial del minimalismo, no es un espacio vacío que daba llenarse sino un área que separa contenidos y ordena visualmente.

- Es adaptable. El minimalismo diseña en torno al contenido: el contenido es el rey. La idea es hacer que el mensaje sea más claro no solo eliminando las distracciones y ambigüedades, sino también manteniendo el enfoque en el contenido. La variedad de dispositivos desde los que puede accederse a los contenidos obliga a que la interfaz sea adaptable (responsive), menos elementos en la pantalla también significa menos datos a procesar en el back-end, lo que acelera los tiempos de carga en la navegación.

El mayor problema al que se enfrenta el diseño plano en las interfaces de usuario plana son los elementos interactivos. El usuario necesitan saber qué elementos de la página son estáticos, y cuáles son accesibles. Esta teoría fue respaldada por Jakob Nielsen, uno de los ingenieros más reputados en el ámbito de la usabilidad, que criticó la simplicidad de diseño de Windows 8. En estos términos, el estudio de Burmistrov, Zlokazova1, Izmalkova y Leonova (2015) mediante la tecnología eye-tracking, demostró que el diseño plano presentaba además mayores dificultades para el usuario. Las búsquedas, el análisis y el procesamiento de los objetos en la interfaz con diseño plano además de llevar más tiempo induce a una tasa de error significativamente mayor. Es así como los autores justifican el necesario retorno a interfaces basadas en principios de diseño tradicionales en la ingeniería de la usabilidad.

En 2014 surgió una interpretación más madura y equilibrada del diseño plano, el diseño flat 2.0. Para Babich (2017), este diseño toma los mejores aspectos del minimalismo y del esqueumorfismo y los hace trabajar juntos. Utiliza sombras sutiles y efectos suaves en los bordes para comunicar interactividad. Cambios sutiles, una evolución natural del diseño (Allen, 2014). No se trata de rebelarse contra las tendencias actuales, sino de mejorar esas tendencias con formas y gradientes simples, es una evolución no una revolución. Las características gráficas de esta evolución para Isabel $(2015)^{7}$ son:

Recurso electrónico disponible en: https://bit.ly/2U4FoGQ 
- Long Shadow: sombras alargadas para añadir dramatismo e interés.

- Leves Degradados: Añaden algo de realismo al elemento.

- Sombras paralelas: aportan volumen al elemento con respecto al fondo.

- Flat Design 3D: Preservando la esencia de la estética del diseño plano.

- Perfiles sutiles: suaves relieves y perfiles que dan volumen a los elementos.

A pesar de las diferencias que parecen evidentes entre Esqueumorfismo y Flat Design, ciertamente no siempre se presentan como técnicas de diseño que están en oposición dialéctica. Tal y como afirma Curtis (2015) los discursos que proponen estas dos técnicas han sido superficialmente interpretados. A través de un examen teórico, el autor desvela que ambas técnicas usadas por las interfaces de Apple, en realidad se basan en las mismas estrategias retóricas, atraer emocionalmente a los usuarios. Ambas técnicas de diseño fueron capaces de convencer a los usuarios de que sus interfaces eran el medio más útil para realizar tareas en su vida cotidiana.

\section{Descripción metodológica}

El diseño metodológico elegido para este estudio es de cualitativo y contribuye al propósito de informar con objetividad, claridad y precisión acerca de una realidad social, en este caso a partir del análisis documental (Rodríguez-Gómez, Gil-Flores y García Jiménez, 1996). Para la comprobación de las hipótesis se ha elegido un modelo de investigación social de tipo observacional, longitudinal y retrospectiva.

Para el estudio de la posible influencia de la Bauhaus en la identidad visual gráfica de las organizaciones, se ha tomado como referencia el ranking de las 100 empresas con mayor reputación en el mundo, Global RepTrack ${ }^{\circledR} 100$, elaborado por el Reputation Institute ${ }^{8}$. Existen otros ranking como el BrandZ $Z^{9}$ elaborado conjuntamente por Kantar Millwardbrown y la multinacional WPP o el de la compañía Interbrand $^{10}$. El Global RepTrack ${ }^{\circledR}$ mide la reputación de más de 7.000 compañías, en 55 países y de 20 sectores productivos diferentes. El matiz que ha motivado la elección de este ranking tiene que ver con el uso del concepto de reputación. Para Villafañe (2001) la reputación corporativa exige una visión integradora porque implica una triple dimensión: axiológica, en la que se encierren los valores culturales de la organización; un comportamiento corporativo comprometido a lo largo del tiempo con relación a los clientes, empleados y accionistas y una actitud proactiva de la organización en la gestión y en la comunicación. La reputación corporativa es la base de un nuevo concepto de liderazgo que ya no se basa en las condiciones clásicas que hasta hace poco eran exigibles en las organizaciones líderes (2001:2).

Este ranking se ha completado con otros casos aparecidos de compañías renombradas. La muestra final se compone de 32 compañías que representan a los principales sectores económicos e industriales: Tecnología y comunicación; Alimentación y bebidas: Automoción; Servicios bancarios y financieros; Entretenimiento; Moda y otros servicios profesionales.

El análisis de la evolución en la identidad visual de las organizaciones se ha realizado mediante consultas en las páginas web corporativas de las mismas, comple-

\footnotetext{
Recurso electrónico disponible en: https://www.reputationinstitute.com

Recurso electrónico disponible en: https://bit.ly/2WW4Ft6

10 Recurso electrónico disponible en: https://bit.ly/2IClNtn
} 
mentado con la información obtenida de sitios web profesionales dedicados al diseño gráfico y la identidad. Se contemplan únicamente los cambios en la identidad más recientes y que son significativos en nuestro estudio.

Las marcas gráficas elegidas fueron trasladadas a una tabla Excel (fig. 6) ordenada de forma descendente en la que se muestran de forma concisa las variaciones que resultan significativas para nuestro estudio. Para la realización del análisis comparativo y las posibles influencias de la Bauhaus y la identidad de las empresas, se realizó además una revisión bibliográfica en torno a las características, peculiaridades y propuestas de la Escuela en el ámbito del diseño gráfico, la publicidad y la identidad visual.

Fig.6. Círculo cromático de Itten.

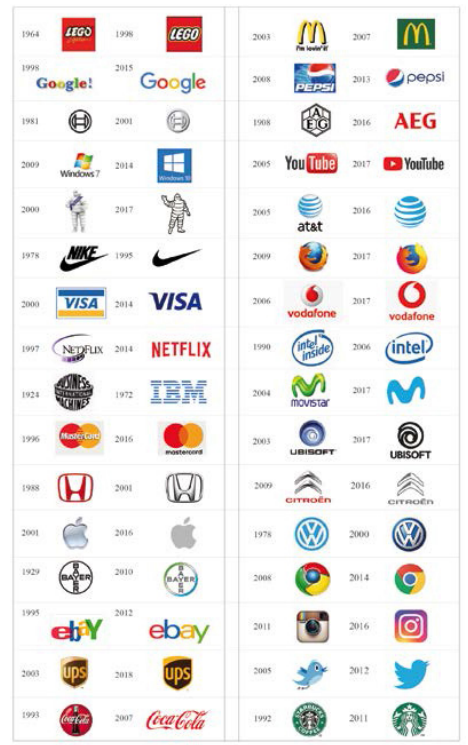

Fuente: elaboración propia

\section{Resultados}

En el puesto número uno de este ranking se sitúa la compañía danesa Lego. Entre 1934 y 1955 la identidad gráfica sufrió importantes transformaciones; desde una imagen plana y sintética en los inicios que recuerda al trabajo de Peter Behrens para AEG, hasta algunos experimentos tridimensionales y la incorporación del tono amarillo a mediados delos cincuenta. Desde ese momento la imagen no se ha visto alterada significativamente, conserva su composición en caja alta con una familia tipográfica redondeada y algo infantil, bordeada con un contorno negro y amarillo sobre un plano de color rojo sólido. La marca no acometió un viaje hacia el esqueumorfis$m o$ y por lo tanto no ha requerido de un regreso al diseño plano.

La compañía IBM constituye un ejemplo paradigmático. El logotipo creado por Paul Rand en 1972, se ha mantenido intacto hasta nuestros días, en lo que podría entenderse como un trabajo visionario de flat design.

AEG podría ser considerada como la primera identidad visual gráfica de la historia, en términos formales. Es muy discutible que la marca gráfica de esta 
compañía alemana fundada en 1883 sea el primer identificador comercial conocido, más si cabe al tener en cuenta otros identificadores como el de Coca-Cola, el Bibendum de Michelín o incluso la Cruz del cristianismo. El caso de AEG sí configura uno de los primeros intentos por formalizar técnica, constructiva y formalmente la implantación de una identidad visual gráfica en una organización. El isologo (texto e icono fundidos en un solo elemento) diseñado por Peter Behrens en 1908 sufrió el cambio más significativo en 1996 se redujo a un logotipo (formaciones de palabras) que además incorporó el color rojo como identificador de la compañía.

El sector automovilístico es uno de los sectores más cambiantes en términos de identidad visual. Encontramos ejemplos de compañías que han evolucionado su identidad para adaptarla a las tendencias esqueumorfistas durante la década de los noventa y principios de dosmil y que poco después regresaron al diseño plano y otras que aún no ha descartado recursos como el biselado y el aspecto metálico y tridimensional. Citroën es un claro ejemplo de restyling ${ }^{I l}$ hacia el flat design en 2016. Un movimiento que han realizado otras marcas como Renault, Honda, Seat o Audi. El mítico rombo de Renault creado por Jean Pierre Vasarelly en 1925 fue renovado en 2004 para aportar el aspecto biselado y metálico que aún hoy conserva. El blitz (rayo en alemán) que caracteriza a Opel desde 1963 se convirtió entre 2002 y 2006 en una figura tridimensional con esquinas oblicuas y una silueta dinámica. En 2016 el isologo regresó a las formas planas. El imagotipo (conjunto icónicotextual) de Honda se creó en 1963 para diferenciar la gama de vehículos de las motocicletas. El icono formado por la inicial de la marca encerrada en un cono truncado invertido conoció a principios de dosmil cambios que le aportaron tridimensionalidad y aspecto metálico. Hoy en día este efecto se ha sustituido por una construcción plana y monocromática. El logotipo inicial de Seat en 1950 con claras influencias heráldicas y la referencia obligada al grupo Fiat evolucionó hasta 1992 dentro de los parámetros clásicos del diseño plano y monocromático. En 1999 y fruto de un cambio de orientación y posicionamiento de marca, la identidad incorporó el color rojo predominante y el biselado metálico de la inicial. Hoy, se usa indistintamente la versión plana y la tridimensional. El concepto inicial de los cuatro anillos en el icono del logotipo de Audi para representar la fusión de cuatro fabricantes alemanes independientes, fue recuperado en 1985 incorporando en los anillos un efecto de bisel redondeado.

El caso de Ford es interesante ya que en 1966 Henry Ford II rechazó el rediseño que realizó Paul Rand en 1966 (Gil, 2018) y que paradójicamente podría englobarse dentro de lo que hoy denominamos flat design (figura 2). El Bibenbum (hombre Michelin) constituye otro ejemplo de esto mismo, esta vez aplicado al ámbito de las mascotas asociadas a una marca.

Observamos un grupo de compañías que han renovado su identidad no para desproveerla de efectos tridimensionales sino para llevar a cabo un proceso de simplificación en relación a la tendencia responsive. Es el caso de Nike, cuyo icono (Swoosh) ha pasado a funcionar de manera independiente con respecto al nombre. Algo parecido ocurrió con la red social Twitter que vio como su mascota Larry the Bird se

11 El término restyling se emplea en diseño gráfico para referirse a la renovación de la identidad gráfica sin afectar a principios, valores o cultura de la marca. Suele afectar a las tipografías, la construcción, el envasado y en menor medida a la paleta cromática empleada. 
simplificaba convirtiéndose en una imagen icónica y desprovista del realismo que apuntaba en sus primeras versiones. Starbucks optó por reducir la imagen del grabado nórdico, por un primer plano de la sirena de dos colas reproducida en el color verde corporativo. El logotipo de Netflix también sufrió un restyling con vistas a simplificar bruscamente la imagen gráfica eliminado la representación icónica de la película cinematográfica y sustituyendo la tipografía de caracteres romanos por una fuente sans-serif y en color rojo.

En el grupo de compañías de alimentación y bebidas el cambio más significativo lo observamos en McDonalds. Los Arcos Dorados (Golden Arches) que caracterizan a la compañía y que podemos definir como isotipo (la parte simbólica o icónica de las marcas), fueron incorporados en 1968, provenientes de la propia decoración de los restaurantes. La unión de los dos arcos genera una imagen que se asemeja a la inicial del nombre. En 2010 la compañía incorporó el color verde para sustituir al clásico rojo que caracteriza a gran parte de las marcas de comida rápida, con la intención de mostrarse como una compañía comprometida con el medio ambiente. En este caso podríamos discutir sobre la pertinencia de definir este cambio como una evolución o como la generación de una submarca. Actualmente, el imagotipo (isotipo+logotipo) conserva el tono dorado de los arcos pero ha eliminado cualquier efecto tridimensional o resaltado y siempre sobre el color verde.

La identidad de Coca-Cola ha permanecido prácticamente inalterable más de un siglo de vida. Tan solo ha incorporado pequeñas revisiones conforme a los gustos o tendencias de la época. El que posiblemente sea hoy el logotipo más reconocible de la historia, no es muy diferente a cómo fue concebido en 1886. El uso de la tipografía Spencerian de inspiración gótica y aspecto caligráfico, unida al color rojo intenso característico de la marca, ha conocido pocos retoques que no han hecho más que homogeneizar los contornos y dotar de más personalidad si cabe a la marca. En 1969 se incorporó a la identidad la Dynamic Ribbon, una especie de ola que añade dinamismo al conjunto gráfico y además recuerda al contorno de la famosa botella Contour patentada por la marca. Durante los años noventa y principios de dos mil, se añadieron diferentes efectos a la identidad para dotarla de mayor profundidad; se emplearon sobras, brillos y elementos corpóreos que aportaban un efecto resaltado. En 2007 la compañía desecha todos estos efectismos y presenta un retorno a la imagen básica y plana, tal y como fue concebida.

La marca gráfica Pepsi es posiblemente la antítesis de Coca-Cola. Creado en 1989 con unas formas muy similares a las de su competidor (tipografía gótica, color rojo, diseño plano). En la década de los cuarenta se renueva para incorporar el azul como referencia directa a los colores de bandera estadounidense, en una muestra de patriotismo durante la Segunda Guerra Mundial. En los sesenta la empresa decidió reformar completamente la identidad gráfica sustituyendo la tipografía inicial por una fuente de palo seco (sans-serif) que se asociaba al contorno de la chapa redonda de las botellas. En la década de los noventa el icono de la chapa, adquiere una forma más compacta (Pepsi Globe) convirtiéndose junto al nombre en un imagotipo. Los efectos de profundidad, resaltado, brillo y tridimensionalidad comenzaron en 1998 . Los siguientes cambios siguieron ahondando en ese estilo tridimensional que persistiría hasta 2013, año en que la compañía afrontó uno de los restyling más significativos: la construcción del icono en plano y la composición del texto en caja baja (minúscula). 
Las compañías tecnológicas han liderado en las dos últimas décadas algunas de las tendencias gráficas en el diseño de la identidad visual. El caso de Google, situada en el segundo puesto del ranking, es un ejemplo singular ya que fue concebida en 1997 bajo algunos parámetros clásicos del esqueumorfismo. La forma actual data de 2015 cuando además de abandonar cualquier referencia al volumen, se cambio la tipografía de estilo clásico hacia una familia más geométrica y simplificada. Los colores se vieron alterados también levemente para mostrar una paleta de tonalidades más básicas y primarias; azul, rojo, amarillo y verde.

Las trayectorias de los otros dos gigantes informáticos, Windows y Apple, ha transcurrido de forma similar. Windows presentó la imagen renovada de su sistema operativo Windows 7 en 2009 con un claro espíritu esqueumorfista. Apple mostró una renovación de la icónica manzana en 2001 como un reflejo de la profunda renovación de la interfaz grafica del sistema. Toda la iconografía y los elementos gráficos del sistema fueron renovados siguiendo las pautas del esqueumorfismo con el objetivo de ofrecer un entorno más realista y familiar para el usuario. En ambos casos, tanto en Windows como en Apple, el viaje al esqueumorfismo terminó por un regreso al más puro flat design. Estas transformaciones pueden apreciarse en otras compañías de base tecnológica como ATT, Vodafone, Movistar, Ubisoft, Chrome, Instagram, Ebay o Youtube.

El caso del buscador Firefox es una excepción ya que si bien durante sus primeros años de vida siempre mantuvo una identidad de aspecto tridimensional, a día de hoy no ha renunciado completamente a un cierto aspecto volumétrico del famoso Firebird (pájaro de fuego), rodeando al globo terrestre. Este ejemplo podríamos englobarlo dentro de la tendencia flat 2.0. Al igual que la compañía Bayer, ninguna de las dos empresas han desprovisto a sus marcas gráficas de sombreados de color y efectos.

En el puesto 10 del ranking se sitúa la compañía Mastercard. El último rediseño de la identidad corrió a cargo de la empresa Pentagram en 2016. Como la propia empresa alude, se realizó para reflejar la disposición y optimismo sobre el futuro, a través de una marca, simplificada, modernizada, y optimizada para su uso en contextos digitales. El entrelazado de las dos esferas de color se simplificó para eliminar ruido gráfico y ganar claridad en los soportes digitales. El concepto de inclusión y accesibilidad que presentaba el símbolo original, evolucionó hacia una mayor concreción. La denominación, situada sobreimpresa sobre el símbolo se emancipó de este para hacerse más visible mediante trazos aún más geométricos en caja baja.

La evolución de su competidor Visa es similar, la marca había sido concebida como un logo-símbolo que recordaba unívocamente al producto (tarjeta). En 2014 esta asociación gráfica se descartó, posiblemente porque el nivel de conocimiento de marca por el público ya era suficiente, y se optó por reducir la identidad a la denominación compuesta con la misma tipografía. Se había eliminado el color dorado de la identidad (símbolo de la reserva de oro estadounidense) y se optó por variar levemente el azul corporativo incluyendo una degradado de color que le diera cierta luminosidad al texto.

Este proceso de simplificación conservando las claves gráficas iniciales también puede observarse en la marca Intel. La incorporación de la palabra inside a la identidad en la década de los ochenta fue descartada en 2006 para regresar a la forma más simplificada y básica. 


\section{Conclusiones}

A partir de la revisión bibliográfica realizada, podemos constatar paralelismos entre algunos conceptos sobre el diseño en la Bauhaus y las tendencias actuales en diseño gráfico, más concretamente el denominado flat design. La ideología de la forma, unida a una percepción novedosa del color y un tratamiento específico de las tipografías generó un estilo singular y unas formas de expresión en la Escuela, que pueden conectar con formas actuales de articulación del diseño gráfico en lo que se refiere al diseño de la identidad de las organizaciones. En la respuesta de la Bauhaus frente a la ornamentación y el naturalismo imperante de la época, encontramos un paralelismo significativo con la ruptura que recientemente ha supuesto el flat design con respecto a la estética gráfica inmediatamente anterior, el denominado esqueumorfismo.

Esta conexión se evidencia con el análisis de la evolución en la identidad visual gráfica de algunas de las marcas más renombradas a nivel mundial durante los últimos años. Durante la década de los noventa y principios de dos mil, la irrupción de las TIC generó un nuevo ecosistema digital mediático, amplificado por nuevos dispositivos y pantallas. Las organizaciones y sus marcas tuvieron que adaptar sus estrategias y modos de expresión a este nuevo contexto integrado por usuarios que no habían sido alfabetizados en las TIC. La solución estaba en el esqueumorfismo, un tratamiento gráfico que a través del realismo conseguía dotar de cercanía y familiaridad a los elementos que integraban las nuevas interfaces digitales. La identidad visual gráfica de las organizaciones se movilizó en esa misma dirección y motivada también por el desarrollo que experimentó el software de edición digital, transformó en gran medida su identidad para mostrar una cara diferente. Es la época del realismo, los brillos, los efectismos, la tercera dimensión simulada y el realismo.

La ruptura con el esqueumorfismo se produjo una vez superada la fase de adaptación al nuevo entorno. La función vuelve a prevalecer sobre la forma, las formas se simplifican, los colores se muestran sólidos, no hay tres dimensiones sino dos, vuelve la «ideología de lo plano». La funcionalidad ahora se relaciona con la adaptabilidad de los contenidos. Los elementos gráficos deben adaptarse a una nueva funcionalidad: provocar una experiencia de usuario simple y fácil, un acceso a los contenidos rápido y eficaz, una limpieza generalizada de la interfaz que elimine ruido innecesario en un contexto saturado de mensajes y contenidos. Este movimiento, como hemos visto se ha evidenciado en gran medida sobre todo en el sector de las empresas tecnológicas, cuya visión y misión empresarial se encuentra más sometida a las fluctuaciones de estilos, a las tendencias y cambios. Aquellas marcas que representan a productos o servicios de consumo masivo, de alguna forma sucumbieron a este viaje de ida y vuelta del que hablamos. Por otra parte, al igual que ocurrió con las voces discordantes o no alineadas completamente con la ideología de la Bauhaus, el flat design encuentra en el flat 2.0. una respuesta a la uniformidad flat.

A modo de conclusión y resumen del estudio, hacemos un ejercicio de esquematización para mostrar la pervivencia de la Bauhaus en la articulación del diseño gráfico actual, concretamente en lo que se refiere a la creación de la identidad visual de las organizaciones y la tendencia conocida como flat design. 
Tabla 1. Bauhaus y flat design.

\begin{tabular}{|l|l|}
\hline \multicolumn{1}{|c|}{ Bauhaus } & \multicolumn{1}{c|}{ Flat Design } \\
\hline $\begin{array}{l}\text { En su origen, plantea una oposición al } \\
\text { Naturalismo y el Art Nouveau; la ornamentación } \\
\text { y los fines puramente decorativos. }\end{array}$ & $\begin{array}{l}\text { Surge como una alternativa al esqueumorfismo, } \\
\text { una tendencia al perfeccionamiento de las formas } \\
\text { por encima de la función. }\end{array}$ \\
\hline Se huye de cualquier referencia a la realidad. & $\begin{array}{l}\text { Al contrario que el esqueumorfismo no intenta ser } \\
\text { realista sino funcional. }\end{array}$ \\
\hline $\begin{array}{l}\text { Las tipografías no son elementos ornamentales } \\
\text { ni deben destacar por su forma, deben facilitar } \\
\text { la lectura. Se emplean tipos sans-serif, } \\
\text { basados en formas geométricas simples }\end{array}$ & $\begin{array}{l}\text { Las tipografías sencillas sin remates ni } \\
\text { ornamentos facilitan la lectura en la interfaz } \\
\text { de los diferentes dispositivos desde los que se } \\
\text { accede a la información. Se busca la claridad y el } \\
\text { minimalismo. }\end{array}$ \\
\hline $\begin{array}{l}\text { El color se entiende también desde su } \\
\text { capacidad de provocar emociones y } \\
\text { sensaciones. Se recurre a paletas cromáticas } \\
\text { básicas de colores primarios. }\end{array}$ & $\begin{array}{l}\text { Se buscan colores sólidos y a ser posible básicos. } \\
\text { Hay una tendencia a eliminar los matices y a no } \\
\text { alterar los tonos con brillos o efectos de luz. }\end{array}$ \\
\hline $\begin{array}{l}\text { El estilo plano prevalece en la pintura y } \\
\text { el diseño, no se busca el volumen sino la } \\
\text { distribución de los elementos en el plano. }\end{array}$ & $\begin{array}{l}\text { Los elementos no tienen volumen, no están } \\
\text { resaltados ni buscan la profundidad. Se trabaja } \\
\text { preferentemente en 2 dimensiones. }\end{array}$ \\
\hline $\begin{array}{l}\text { Las propuestas se adaptan a la función. La } \\
\text { función prevalece sobre la forma. No se busca } \\
\text { el placer estético. }\end{array}$ & $\begin{array}{l}\text { El diseño se genera pensando en su adaptabilidad, } \\
\text { su capacidad de mostrarse con coherencia y } \\
\text { uniformidad en diferentes soportes, pantallas, } \\
\text { dispositivos, etc. }\end{array}$ \\
\hline $\begin{array}{l}\text { Hay una tendencia a la simplificación en torno } \\
\text { a las } 3 \text { formas básicas: el cuadrado, el círculo y } \\
\text { el triángulo. En la Escuela también hubo voces } \\
\text { discordantes como la de Oskar Schlemmer que } \\
\text { no aceptaban del todo el dogmatismo en la } \\
\text { ideología de lo plano }\end{array}$ & $\begin{array}{l}\text { El flat 2.0, como evolución del flat design } \\
\text { incorpora leves elementos tridimensionales o } \\
\text { realistas (sombras, brillos, relieves). }\end{array}$ \\
\hline
\end{tabular}

Fuente: Elaboración propia

\section{Referencias bibliográficas}

Abbott, E. A. (1999). Planilandia. Una novela de muchas dimensiones. Mallorca: José J. de Olañeta.

Allen, R. (2014): «Flat Design Has Evolved», Medium, https://medium.com/@ryanallen_ com/flat-design-has-evolved-7f396a541953. Web visitada el 24/01/2019.

Babich, N. (2017): «Best Practices for Flat Design», Uxplanet, https://uxplanet.org/bestpractices-for-flat-design-6e7a6997805. Web visitada el 13/02/2019.

Balmer, J. (2001): «Corporate Identity, Corporate Branding and Corporate Marketing: Seeing through the Fog», European Journal of Marketing, 35(3/4), 248- 291. DOI: 10.1108/03090560110694763.

Barnes, S. (2007): «Alan Kay: Transforming the Computer into a Communication Medium», IEEE Annals of the History of Computing, 29(18-30). DOI: 10.1109/MAHC. 2007. 17 
Burmistrov, I., Zlokazova, T., Izmalkova, A., y Leonova, A. (2015): «Flat Design vs Traditional Design: Comparative Experimental Study», en Abascal, J. et al. (Eds.): Interact 2015, Part II, LNCS 9297, 106-114, DOI: 10.1007/978-3-319-22668-2_10.

Cao, J., Zieba, K., Ellis, M. y Stryjewski, K. (2015): «Elegant Wev UI Design Techniques: Flat Design \& Colors», UXPin, https://www.uxpin.com/studio/ebooks/web-ui-designtechniques-colors-flat-design/. Web visitada el 08/12/2018.

Chaves, N. y Bellucia, R. (2003): La marca corporativa. Gestión y diseño de símbolos y logotipos. Buenos Aires: Paidós.

Contreras, F. y San Nicolás, C. (2001): Diseño gráfico, creatividad y comunicación. Madrid: Blur Ediciones.

Costa, J. (1987): Imagen global. Evolución del diseño de identidad. Barcelona: Ceac.

Currás Pérez, R. (2010): «Identidad e imagen corporativas: revisión conceptual e interrelación», Teoría y Praxis, 7, 9-34.

Curtis, A. (2015): Rhetoric of flat design and skeuomorphism in Apple's iOS graphical user interface, Tesis de Maestría, 638, http://digitalcommons.uri.edu/theses/638, Web visitada el 18/06/2019.

Drosde, M. (1993): Bauhaus (1919-1933). Berlín: Benedikt Taschen Verlag GmbH.

Frutiger, A. (2007): Signos, símbolos, marcas, señales. Elementos, morfología, representación, significación. Barcelona: Gustavo Gili.

Gil, J. (2018): «Cuando Henry Ford le dijo no a Paul Rand», https://graffica.info/henry-forddijo-no-a-paul-rand/ Web visitada el 29/01/2019.

Grinshtein, A. (2012): The Flat Design Era, http://layervault.tumblr.com/post/32267022219/ flat-interface-design. Web visitada el 30/01/2019.

Kotler, PH. y Armstrong, G. (1991): Fundamentos del marketing ( $6^{a}$ Edición). México: Pearson Educación.

Mínguez, N. (2000): «Un marco conceptual para la imagen corporativa», Zer, 835(3/4), 292315.

Molina Ruíz, J. L. (2010): «25 años de la revolución de los iconos en el escritorio». I+Diseño: revista internacional de investigación, innovación y desarrollo en diseño, 2(2), 13-17.

Mouzon, S. A. (2010): The Original Green: Unlocking the Mystery of True Sustainability. Miami: Guild Foundation Press.

Pelta, R. (2012-A): «Paul Rand. Comunicar con ingenio». Monográfica, 3. http://www.monografica.org/03/Art\%C3\%ADculo/4562. Web visitada el 13/12/2018.

- (2012-B): «El pensamiento tipográfico moderno», Monográfica.org, 4, http://www.monografica.org/04/Art\%C3\%ADculo/5824. Web visitada el 13/12/2018.

Rodríguez-Gómez, G., Gil-Flores, J. y García Jiménez, E. (1996): Metodología de la investigación cualitativa. Granada: Ediciones Aljive.

Rodríguez González, A. (2005): ¿Logo qué? México D.F.: Siglo XXI Editores.

Sanz de la Tajada, L. A. (1996): Auditoría de la imagen de empresa. Métodos y técnicas de estudio de la imagen. Madrid: Síntesis.

Vadillo, M. (2016): Las diseñadoras de la Bauhaus: Historia de una revolución silenciosa. Sevilla: Editorial Cántico.

Villafañe, J. (2001): «La reputación corporativa como factor de liderazgo», Área Abierta, 1(4).

- (1998): Imagen positiva. Gestión estratégica de la imagen de las empresas. Madrid: Pirámide.

Witford, F. (1991): La Bauhaus. Barcelona: Ediciones Destino.

Wick, R. (1982): La pedagogía de la Bauhaus. Madrid: Alianza. 